Pacific Journal of Mathematics

ON THE STABILITY OF THE SET OF EXPONENTS OF A 


\section{ON THE STABILITY OF THE SET OF EXPONENTS OF A CAUCHY EXPONENTIAL SERIES}

\section{S. VERBLUNSKY}

If $f \in L(-D, D)$ and $Q(z)$ is a meromorphic function whose poles, all simple, forms a sub-set of the set $\left\{\lambda_{\nu}\right\}(\nu=0, \pm 1$, $\pm 2, \cdots)$, then the C.E.S. (Cauchy exponential series) of $f$ with respect to $Q(z)$ is $\Sigma c_{\nu} e^{\lambda_{\nu} x}$, where

$$
c_{\nu} e^{\lambda_{\nu} x}=\underset{\lambda_{\nu}}{\operatorname{res}} Q(z) \int_{-D}^{D} f(t) e^{z(x-t)} d t .
$$

Suppose we are given a class $A$ of functions $f$ each of which can be 'represented' in $(-D, D)$ by its C.E.S. with respect to $Q(z)$. We define a set of neighbourhoods $U$ of $\left\{\lambda_{\gamma}\right\}$. Then $\left\{\lambda_{\gamma}\right\}$ is stable if there is a $U$ such that to each $\left\{\kappa_{\nu}\right\} \in U$ there corresponds a meromorphic function $q(z)$ whose poles, all simple, form a sub-set of $\left\{\kappa_{\nu}\right\}$ and which is such that each $f \in A$ can be represented in $(-D, D)$ by its C.E.S. with respect to $q(z)$; and $\left\{\lambda_{y}\right\}$ is unstable if there is no such neighbourhood.

The case in which $\lambda_{\nu}=i \nu, A$ is $B V[-D, D]$, 'representation of $f$ in $(-D, D)$ ' means ' $\sum_{|\nu| \leqq n} c_{\nu} e^{\lambda_{\nu} x} \rightarrow 1 / 2(f(x+)+f(x-))$ boundedly within $(D, D)$ ' is considered. It is shown, in particular, that with reasonable conditions on the set of neighbourhoods $U,\{i \nu\}$ is unstable if $D>1 / 2 \pi$, and stable if $D=1 / 2 \pi$.

Let $D>0$ and $f \in L(-D, D)$. I et $Q(z)$ be a meromorphic function whose poles, all simple, form a sub-set of the set $\left\{\lambda_{\nu}\right\}(\nu=0, \pm 1, \cdots)$. Here, and in what follows, the use of the symbol $\left\{\lambda_{\nu}\right\}$ implies that $\lambda_{\nu} \neq \lambda_{\nu}$, if $\nu \neq \nu^{\prime}$. The C. E. S. (Cauchy exponential series) of $f$ with respect to $Q$ is $\sum c_{\nu} e^{\lambda_{\nu} x}$ where

$$
c_{\nu} e^{\lambda_{\nu} x}=\underset{\lambda_{\nu}}{\operatorname{res}} Q(z) \int_{-D}^{D} f(t) e^{z(x-t)} d t .
$$

Suppose that the set $\left\{\lambda_{\nu}\right\}$ is such that, for a class $A$ of functions $f$, the C.E.S. of $f$ 'represents' $f$ in $(-D, D)$. Then we may consider the question of the stability of the set $\left\{\lambda_{\nu}\right\}$. We define, in some way, a set of neighbourhoods $U$ of $\left\{\lambda_{\nu}\right\}$. Then $\left\{\lambda_{\nu}\right\}$ is stable if there is a neighbourhood $U$ such that to each $\left\{\kappa_{\nu}\right\} \in U$, there corresponds a meromorphic function $q(z)$ whose poles, all simple, form a sub-set of $\left\{\kappa_{\nu}\right\}$, and which is such that each $f \in A$ can be represented in $(-D, D)$ by its C.E.S. with respect to $q(z)$; and $\left\{\lambda_{\nu}\right\}$ is unstable if there is no such neighbourhood. The stability of $\left\{\lambda_{\nu}\right\}$ depends on the value of $D$, the class $A$, the, particular meaning we give to the 'representation' of $f$,

Received July 27, 1964. 
and finally on the definition of the set of neighbourhoods $U$. In this note, we confine our attention to the simplest case: $\lambda_{\nu}=i \nu, A$ is the class of functions $f$ which are $B V[-D, D]$ and satisfy $2 f(x)=$ $f(x+)+f(x-)$ in $(-D, D)$, 'representation' of $f$ means 'bounded convergence to $f(x)$ within $(-D, D)$ ', i.e., for each $\delta$ satisfying $0<\delta<D, \sum_{|\nu| \leqq n} c_{\nu} e^{\lambda_{2} x} \rightarrow f(x)$ boundedly in the segment $|x| \leqq D-\delta$. We recall that if $D=\pi$, then each $f \in A$ can be represented by its C.E.S. with respect to $Q_{0}(z)=1 / 2 \operatorname{coth} \pi z$, since, in this case, the C.E.S. is the Fourier series of $f$. Let us suppose that to each neighbourhood $U$ there corresponds an $\varepsilon>0$ such that $\left\{\mu_{\nu}\right\} \in U$ if $\sum\left|\mu_{\nu}-\lambda_{\nu}\right|<\varepsilon$; and to each $\delta>0$ there corresponds a neighbourhood $U_{\delta}$ such that if $\left\{\mu_{\nu}\right\} \in U_{\delta}$ then $\sup \left|\mu_{\nu}-\lambda_{\nu}\right|<\delta$. What we prove, implies that $\{i \nu\}$ is unstable if $D>\pi / 2$, and stable if $D=\pi / 2$. We shall, however, prove more than this, viz.

THEOREM 1. Let $\left\{l_{\nu}\right\}$ be a real set not containing every integer, such that $l_{\nu}$ is an integer for $|\nu| \geqq N$. If $D>\pi / 2$, then there is no meromorphic function $q(z)$ whose poles, all simple, form a sub-set of $\left\{i l_{\nu}\right\}$ and which is such that each $f \in A$ can be represented by its C.E.S. with respect to $q$.

THEOREM 2. Let $l_{\nu}=\nu+\alpha_{\nu}+i \beta_{\nu}$ where $\alpha_{\nu}, \beta_{\nu}$ are real numbers which satisfy

$$
\varlimsup_{|\nu| \rightarrow \infty}\left|\alpha_{\nu}\right|<\frac{1}{8}, \quad \varlimsup_{|\nu| \rightarrow \infty}\left|\beta_{\nu}\right|<\infty .
$$

If $D=\pi / 2$, there exists a meromorphic function $q(z)$ whose poles, all simple, form a sub-set of $\left\{i l_{\nu}\right\}$ and which is such that each $f \in A$ can be represented by its C.E.S. with respect to $q$.

THEOREM. 3. The conclusion of Theorem 2 holds if the condition on $\alpha_{\nu}$ is replaced by $\sup \left|\alpha_{\nu}\right|<1 / 4$.

The relation between Theorem 2 and the work of Korous [1] is explained in $\S 6$. The relation between Theorem 3 and the work of Levinson [2] is explained in $\S 7$.

2. Let $0<D \leqq \pi$, and let $A$ have the meaning specified in $\S 1$.

ILEMMA 1. If $H_{n}(t) \in L(-2 D, 2 D)$ for $n \geqq n_{0}$, then, in order that for each $f \in A$,

$$
\int_{-D}^{D} f(t) H_{n}(t-x) d t \rightarrow f(x)
$$


boundedly within $(-D, D)$, it is necessary and sufficient that

$$
\int_{0}^{t} H_{n}(u) d u \rightarrow \frac{1}{2} \operatorname{sgn} t
$$

boundedly within $(-2 D, 2 D)$.

Proof. Let

$$
J_{n}(u)=\frac{1}{2 \pi} \frac{\sin \left(n+\frac{1}{2}\right) u}{\sin \frac{1}{2} u}
$$

Then for each $f \in A$,

$$
\int_{-D}^{D} f(t) J_{n}(t-x) d t \rightarrow f(x)
$$

boundedly within $(-D, D)$, and

$$
\int_{0}^{t} J_{n}(u) d u \rightarrow \frac{1}{2} \operatorname{sgn} t
$$

boundedly within $(-2 D, 2 D)$. Let $K_{n}(u)=H_{n}(u)-J_{n}(u)$. It suffices to prove: in order that for each $f \in A$,

$$
\int_{-D}^{D} f(t) K_{n}(t-x) d t \rightarrow 0
$$

boundedly within $(-D, D)$, it is necessary and sufficient that

$$
k_{n}(t)=\int_{0}^{t} K_{n}(u) d u \rightarrow 0
$$

boundedly within $(-2 D, 2 D)$.

Sufficiency. We have

$$
\begin{aligned}
\int_{-D}^{D} f(t) K_{n}(t-x) d t & =f(D) k_{n}(D-x)-f(-D) k_{n}(-D-x) \\
& -\int_{-D}^{D} k_{n}(t-x) d f(t)
\end{aligned}
$$

and the second member tends to zero boundedly within $(-D, D)$.

Necessity. In the first place, it is necessary that for each $\tau \in(-2 D, 2 D), k_{n}(\tau) \rightarrow 0$ as $n \rightarrow \infty$. For let $\alpha, \beta \in(-D, D)$ and let $x=\alpha$. Let $f(t)=1$ in the open interval, and let $f(t)=0$ outside the closed interval, whose end points are $\alpha, \beta$. Then 


$$
k_{n}(\beta-\alpha)=\int_{\alpha}^{\beta} K_{n}(t-\alpha) d t \rightarrow 0 .
$$

Since $\alpha, \beta$ can be chosen so that $\beta-\alpha$ has any assigned value in $(-2 D, 2 D)$, this proves our assertion.

By (1), for each $x \in(-D, D)$, the functions $k_{n}(t-x)$ of $t$, for $n \geqq n_{0}$, form a sequence of elements of $C[-D, D]$ such that

$$
\int_{-D}^{D} k_{n}(t-x) d f(t)
$$

is convergent for each $f \in A$. By the principle of uniform boundedness, it follows that

$$
\sup _{t \in[-D, D]}\left|k_{n}(t-x)\right|<\infty \text {. }
$$

Choose $x=D-\delta$. Then $k_{n}(t)$ is uniformly bounded in $[-2 D+\delta, \delta]$. Choose $x=-D+\delta$. Then $k_{n}(t)$ is uniformly bounded in $[-\delta, 2 D-\delta]$. Hence $k_{n}(t)$ is uniformly bounded within $(-2 D, 2 D)$ as required.

3. Proof of Theorem 1. We may suppose that $D \leqq \pi$. Let $\omega$ be chosen to satisfy $\pi<\omega<2 D$. We choose the notation so that if $0 \in\left\{l_{\nu}\right\}$ then $0=l_{0}$. If a meromorphic function $q(z)$, with the properties mentioned in the enunciation, exists, let $C_{n}$ denote a contour which contains in its interior precisely those $i l_{\nu}$ for which $|\nu| \leqq n$, and which does not pass through any of the $i l_{\nu}$. Let

$$
H_{n}(u)=\frac{1}{2 \pi i} \int_{\sigma_{n}} q(z) e^{-z u} d z .
$$

If $\sum c_{\nu} e^{i l_{\nu} x}$ is the C.E.S. of $f$ with respect to $q(z)$, then

$$
\begin{aligned}
\sum_{|\nu| \leqq n} c_{\nu} e^{i l_{\nu} x} & =\sum_{|\nu| \leqq n} \operatorname{res}_{i l_{\nu}} q(z) \int_{-D}^{D} f(t) e^{z(x-t)} d t \\
& =\int_{-D}^{D} f(t) H_{n}(t-x) d t .
\end{aligned}
$$

We have

$$
\begin{aligned}
\int_{0}^{x} H_{n}(u) d u & =\frac{1}{2 \pi i} \int_{o_{n}} q(z) \frac{1-e^{-z x}}{z} d z \\
& =\sum_{|\nu| \leqq n} \frac{r_{\nu}}{i l_{\nu}}\left(1-e^{-i l_{\nu} x}\right)
\end{aligned}
$$

where $r_{\nu}$ is the residue of $q(z)$ at $i l_{\nu}$ and where, if $l_{0}=0$, we use the convention

$$
\frac{1-e^{-i l_{0} t}}{i l_{0}}=\lim _{l \rightarrow 0} \frac{1-e^{-i l t}}{i l}=t
$$


By Lemma 1 , it is necessary that

$$
\sum_{|\nu| \leqq n} \frac{r_{\nu}}{i l_{\nu}}\left(1-e^{-i l_{\nu} x}\right) \rightarrow \frac{1}{2} \operatorname{sgn} x
$$

boundedly within $(-2 D, 2 D)$, and hence in $[-\omega, \omega]$. Let $x \in(-\omega$, $\omega-2 \pi)$. Then for $|\nu| \geqq N$, the terms on the left are unaltered on replacing $x$ by $x+2 \pi$. By subtraction, it follows that

$$
\sum_{|\nu|<N} \frac{r_{\nu}}{i l_{\nu}} e^{-i l_{\nu} x}\left(e^{-i l_{\nu} 2 \pi}-1\right)=-1
$$

for such $x$, and hence for all $x$. We note that if $l_{0}=0$, the term with $\nu=0$ is $-r_{0} 2 \pi$. At this point, we distinguish to cases, (a) $l_{0} \neq 0$, (b) $l_{0}=0$.

In case (a), we integrate (7) over $(-X, X)$, divide by $2 X$, and let $X \rightarrow \infty$. We obtain a contradiction. In case (b), we take mean values as in case (a), and deduce that the term with $\nu=0$ is -1 . Then (7) implies that

$$
\sum_{0<|\nu|<N} \frac{r_{\nu}}{i l_{\nu}} e^{-i l_{\nu} x}\left(e^{-i l_{\nu} 2 \pi}-1\right)=0
$$

for all $x$. If we multiply this by its conjugate, and take mean values, we deduce that

$$
\sum_{0<|\nu|<N} \frac{\left|r_{\nu}\right|^{2}}{l_{\nu}^{2}} \sin ^{2} \pi l_{\nu}=0
$$

By (6),

$$
\sum_{0<|\nu| \leqq n} \frac{r_{\nu}}{i l_{\nu}}\left(1-e^{-i l_{\nu} x}\right) \rightarrow \frac{1}{2} \sin x-\frac{x}{2 \pi}
$$

boundedly within $(-2 D, 2 D)$. Considering odd parts, its follows that

$$
\sum_{0<|\nu| \leqq n} \frac{r_{\nu}}{l_{\nu}} \sin l_{\nu} x \rightarrow \frac{1}{2} \operatorname{sgn} x-\frac{x}{2 \pi}
$$

boundedly within $(-2 D, 2 D)$. By hypothesis, there is an integer $\mu$ say, which is not one of the $l_{\nu}$; and $\mu \neq 0$ since $l_{0}=0$. By (8), $r_{\nu}=0$ if $l_{\nu}$ is not an integer. Hence, on multiplying both sides of (9) by $\mu \sin \mu x$ and integrating over $(-\pi, \pi)$, we obtain $0=1$, a contradiction.

4. Proof of Theorem 2. For all sufficiently large $n$, the circle $\Gamma_{n}:|z|=n+1 / 2$, contains in its interior the points $i l_{\nu}$ for $|\nu| \leqq n$, and every point on $\Gamma_{n}$ is at a distance greater than $3 / 8$ from all the points $i l_{\nu}$. Let $q(z)$ be a meromorphic function whose poles, all simple, 
form a sub-set of $\left\{i l_{\nu}\right)$, and define $H_{n}(u)$ by (2) with $C_{n}$ replaced by $\Gamma_{n}$. Using the notation of $\S \S 1,2$, we have

$$
J_{n}(u)=\frac{1}{2 \pi i} \int_{\Gamma_{n}} Q_{0}(z) e^{-z u} d z,
$$

and therefore, as in $\S 2$, it suffices to prove that we can choose $q(z)$ so that

$$
\int_{0}^{x} K_{n}(u) d u=\frac{1}{2 \pi i} \int_{\Gamma_{n}}\left(q(z)-Q_{0}(z)\right) \frac{1-e^{-z x}}{z} d z \rightarrow 0
$$

boundedly within $(-\pi, \pi)$.

Write

$$
P(z)=\left(z-i l_{0}\right) \prod_{1}^{\infty}\left(1-\frac{z}{i l_{\nu}}\right)\left(1-\frac{z}{i l_{-\nu}}\right) .
$$

In $\S 5$, we shall prove

LemMA 2. As $|z| \rightarrow \infty, P(z)=o\left(|z|^{1 / 2} e^{\pi|r e z|}\right)$. On $\Gamma_{n},|P(z)|^{-1}=$ $o\left(n^{1 / 2} e^{-\pi|r e z|}\right)$ as $n \rightarrow \infty$.

The meromorphic function $Q_{0}(z) P(z)$ is regular, except possibly at the points $i \nu$, which are at most simple poles of residue $P(i \nu) / 2 \pi$. By Lemma 2, $P(i \nu)=o\left(|\nu|^{1 / 2}\right)$. Hence we can define the meromorphic function

$$
R(z)=\frac{1}{2 \pi}\left[\frac{P(0)}{z}+\Sigma^{\prime} P(i \nu)\left(\frac{1}{z-i \nu}+\frac{1}{i \nu}\right)\right]
$$

which has the same principal parts as $Q_{0}(z) P(z)$. Thus

$$
Q_{0}(z) P(z)=R(z)+S(z)
$$

where $S(z)$ is an integral function. We can write $q(z) P(z)=F(z)$, where $F(z)$ is an integral function. Then

$$
q(z)-Q_{0}(z)=\frac{F(z)-S(z)-R(z)}{P(z)}
$$

In $\S 5$, we shall prove

IJEMMA 3. On $\Gamma_{n}, R(z)=o\left(n^{1 / 2}\right)$ as $n \rightarrow \infty$.

We choose $F(z)$ so that the numerator in (10) will not be of a greater order of magnitude than $R(z)$. This means, since $F$ and $S$ are integral functions, that $F=S+c$ where $c$ is a constant. Theorem 2 will follow if we show that 


$$
I_{n}(x)=\int_{\Gamma_{n}} \frac{c-R(z)}{P(z)} \cdot \frac{1-e^{-z x}}{z} d z
$$

tends to zero boundedly within $(-\pi, \pi)$. Write $z=(n+1 / 2) e^{i \theta}$. By Lemmas 2 and 3 ,

$$
\frac{c-R(z)}{P(z)}=o\left(n e^{-n \pi|\cos \theta|}\right) .
$$

If then $|x| \leqq \pi-\delta, \delta>0$, we have

$$
I_{n}(x)=o\left(n \int_{0}^{2 \pi} e^{-n \delta|\cos \theta|} d \theta\right)=o(1) .
$$

5. In order to prove Lemmas 2 and 3 , it will be convenient to write

$$
P(i z)=i p(z)
$$

so that

$$
p(z)=\left(z-l_{0}\right) \prod_{1}^{\infty}\left(1-\frac{z}{l_{\nu}}\right)\left(1-\frac{z}{l_{-\nu}}\right),
$$

and

$$
\begin{aligned}
R(i z) & =r(z) \\
& =\frac{1}{2 \pi}\left[\frac{p(0)}{z}+\Sigma^{\prime} p(\nu)\left(\frac{1}{z-\nu}+\frac{1}{\nu}\right)\right] .
\end{aligned}
$$

We need the following result, which is a special case $(a=0)$ of [3] Theorem 1 (with a change of notation).

Lemma 4. Let $L, M$ be positive numbers. Let $s_{\nu}=\nu+\sigma_{\nu}+i \tau_{\nu}$, where $\sigma_{\nu}, \tau_{\nu}$ are real numbers which satisfy $\left|\sigma_{\nu}\right| \leqq L,\left|\tau_{\nu}\right| \leqq M$ for all $\nu$. Suppose that there is a $\delta>0$ such that $\left|s_{\nu}\right| \geqq \delta$ for all $\nu$. Let

$$
\psi(z)=\left(1-\frac{z}{s_{0}}\right) \prod_{1}^{\infty}\left(1-\frac{z}{s_{\nu}}\right)\left(1-\frac{z}{s_{-\nu}}\right) .
$$

Then there is a positive constant $C$ (depending only on $L, M, \delta$ ) such that,

(i) for all $z,|\psi(z)|<C(1+|z|)^{4 L} e^{\pi|i m z|}$;

(ii) if $\left|z-s_{\nu}\right| \geqq \delta$ for all $\nu$, then $|\psi(z)|^{-1}<C(1+|z|)^{4 L} e^{-\pi|i m z|}$.

Proof of Lemma 2. We can find a positive number $L<1 / 8$ such that $\left|\alpha_{\nu}\right| \leqq L$ for $|\nu|>N$ say; and a positive number $M$ such that $\left|\beta_{\nu}\right| \leqq M$ for all $\nu$. In Lemma 4 , choose $s_{\nu}=l_{\nu}$ for $|\nu|>N$; $=\nu$ for 
$0<|\nu| \leqq N ;=3 / 8$ for $\nu=0$. Then $p(z) / \psi(z)$ tends to a nonzero constant as $|z| \rightarrow \infty$. By Lemma 4 (with $\delta=3 / 8$ ), there is a positive constant $D$ such that

(i) $|p(z)|<D|z|^{4} e^{\pi|i m z|}$ if $|z|$ is sufficiently large;

(ii) if $z$ is on $\Gamma_{n}$ and $n$ is sufficiently large then $|p(z)|^{-1}<D n^{4 L} e^{-\pi|i m z|}$ (the condition $\left|z-s_{\nu}\right| \geqq 3 / 8$ for all $\nu$ being satisfied). Since $P(z)=$ $i p(-i z)$, and $4 L<1 / 2$, the lemma follows.

Proof of Lemma 3. By (i) above, $p(\nu)=O\left(|\nu|^{4 L}\right)$. By (11), it will suffice to prove that if $z$ is on $\Gamma_{n}$, then

$$
\Sigma^{\prime} \frac{z p(\nu)}{\nu(z-\nu)}=o\left(n^{1 / 2}\right)
$$

The left hand side is

$$
O\left[\sum_{0<\nu \leqq n} \frac{n \nu^{4 L}}{\nu\left(n+\frac{1}{2}-\nu\right)}+\sum_{n<\nu \leqq 2 n} \frac{n \nu^{4 L}}{\nu\left(\nu-n-\frac{1}{2}\right)}+\sum_{\nu>2 n} n \nu^{4 L-2}\right] .
$$

The first and second sums are $O\left(n^{4 L} \log n\right)$. The third sum is $O\left(n^{4 L}\right)$. This proves the lemma.

In Lemma 4 , we could replace $4 L$ by $2 L$, if the $\sigma_{\nu}$ satisfy the further condition

$$
\sum_{|\nu| \leqq n} \frac{\sigma_{\nu}}{\nu+\frac{1}{2}}=O(1)
$$

This follows from [3] Theorem 2. Hence, as the preceding proof shows, we can replace $1 / 8$ by $1 / 4$ in Theorem 2 if we add the condition

$$
\sum_{|\nu| \leqq n} \frac{\alpha_{\nu}}{\nu+\frac{1}{2}}=O(1)
$$

6. The function $q(z)$ of $\S 4$ is given by

$$
q(z)=\frac{1}{2} \operatorname{coth} \pi z+\frac{c-R(z)}{P(z)} .
$$

Let

$$
\begin{aligned}
q_{0}(z) & =i q(i z) \\
& =\frac{1}{2} \cot \pi z+\frac{c-r(z)}{p(z)} .
\end{aligned}
$$

If $\sum c_{\nu} e^{i l_{\nu} x}$ is the C.E.S. of $f$ with respect to $q(z)$, then, for all sufficiently large $n$, 


$$
\begin{aligned}
\sum_{|\nu| \leqq n} c_{\nu} e^{i l_{\nu} x} & =\frac{1}{2 \pi i} \int_{\Gamma_{n}} q(z) d z \int_{-\pi / 2}^{\pi / 2} f(t) e^{\sigma(x-t)} d t \\
& =\frac{1}{2 \pi i} \int_{\Gamma_{n}} q_{0}(z) d z \int_{-\pi / 2}^{\pi / 2} f(t) e^{i z(x-t)} d t .
\end{aligned}
$$

Suppose now that $\beta_{\nu}=0$ for all $\nu$, and that $c$ is real. Then $q_{0}(z)$ is real for real $z$, so that $q_{0}(\bar{z})=\overline{q_{0}(z)}$. If

$$
r_{\nu}=\operatorname{res}_{i l_{\nu}} q(z)=\operatorname{res}_{l_{\nu}} q_{0}(z),
$$

then $r_{\nu}$ is real. Let $f$ be real. Write

$$
a_{\nu}-i b_{\nu}=c_{\nu}=r_{\nu} \int_{-\pi / 2}^{\pi / 2} f(t) e^{-i l_{\nu} t} d t
$$

Equating real parts in (12), we get

$$
\sum_{|\nu| \leqq n} a_{\nu} \cos l_{\nu} x+b_{\nu} \sin l_{\nu} x=\frac{1}{2 \pi i} \int_{\Gamma_{n}} q_{0}(z) d z \int_{-\pi / 2}^{\pi / 2} f(t) \cos z(x-t) d t
$$

We thus obtain the class of trigonometric series investigated by Korous [1]. Theorem 2 shows, in this special case, not only that (13) converges boundedly to $f(x)$ within $(-\pi / 2, \pi / 2)$, but also that

$$
\sum_{|\nu| \leqq n} a_{\nu} \sin l_{\nu} x-b_{\nu} \cos l_{\nu} x
$$

converges boundedly to zero.

7. We now turn to the proof of Theorem 3. We again suppose that the notation has been chosen so that if $0 \in\left\{l_{\nu}\right\}$, then $0=l_{0}$. It will suffice to prove

LEmMA 5. Under the conditions of Theorem 3, there are complex numbers $w_{\nu}$ such that

$$
\sum_{|\nu| \leqq n} w_{\nu} e^{\imath_{\nu} x} \rightarrow \frac{1}{2} \operatorname{sgn} x
$$

boundedly within $(-\pi, \pi)$.

For then, by the classical theorem of Mittag-Leffler, there is a meromorphic function $q(z)$ whose poles form a sub-set of $\left\{i l_{\nu}\right\}$, the principal part at $i l_{\nu}$ being $i l_{\nu} w_{\nu} /\left(z-i l_{\nu}\right)$ if $l_{\nu} \neq 0$. If $l_{0}=0$, we allow the origin to be a regular point. Defining $H_{n}(u)$ by $(2)$, we have

$$
\int_{0}^{x} H_{n}(u) d u=\frac{1}{2 \pi i} \int_{\sigma_{n}} q(z) \frac{1-e^{-z x}}{z} d z
$$




$$
=\sum_{|\nu| \leqq n} w_{\nu}\left(1-e^{-i l_{\nu} x}\right)
$$

By Lemma 5,

$$
\sum_{|\nu| \leqq n} w_{\nu} \rightarrow 0, \quad \sum_{|\nu| \leqq n} w_{\nu} e^{-i l_{\nu} x} \rightarrow-\frac{1}{2} \operatorname{sgn} x
$$

boundedly within $(-\pi, \pi)$. Thus, Theorem 3 will follow from Lemma 1 .

One way of proving Lemma 5 is to generalize the following theorem of Levinson $[2,48]$ : if the real numbers $\lambda_{\nu}$ satisfy $\left|\lambda_{\nu}\right| \leqq P<1 / 4$, then there are numbers $w_{\nu}$ such that

$$
\sum_{|\nu| \leqq n}\left[w_{\nu} e^{i \lambda_{\nu} x}-\frac{e^{-i \nu x}}{2 \pi} \int_{-\pi}^{\pi} f(t) e^{-i \nu t} d t\right]
$$

converges uniformly to zero within $(-\pi, \pi)$ if $f \in L^{2}(-\pi, \pi)$. The generalization consists in showing that we can replace the real $\lambda_{\nu}$ by $\nu+\alpha_{\nu}+i \beta_{\nu}$, where $\left|\alpha_{\nu}\right| \leqq P$ and $\varlimsup_{|\nu| \rightarrow \infty}\left|\beta_{\nu}\right|<\infty$. However, we only need the result for the function $f(t)=1 / 2 \operatorname{sgn} t$. It seems worthwhile to prove this special case, for which the argument of Levinson can be given a rather simple form. This is done in $\S 9$.

8. We need the following deduction from Lemma 4 .

Lemma 6. Let $S_{\nu}=\nu+\sigma_{\nu}+i \tau_{\nu}$, where $\sigma_{\nu}, \tau_{\nu}$ are real numbers which satisfy $\left|\sigma_{\nu}\right| \leqq P,\left|\tau_{\nu}\right| \leqq Q$ for all $\nu$, where $0<P<1 / 4$ and $Q>0$. Let

$$
\Psi(z)=\left(z-S_{0}\right) \prod_{1}^{\infty}\left(1-\frac{z}{S_{\nu}}\right)\left(1-\frac{z}{S_{-\nu}}\right) .
$$

Then there is a constant $K$ (depending only on $P$ and $Q$ ) such that

$$
|\Psi(z)|<K(1+|z|)^{4 P} e^{\pi|i m z|} .
$$

and there is a constant $K_{\varepsilon}$ (depending only on $P, Q$ and $\varepsilon$ ) such that

$$
|\Psi(g)|^{-1}<K_{\varepsilon}\left(1+|z|^{4 P} e^{-\pi|i m z|}\right.
$$

if $\left|z-S_{\nu}\right| \geqq \varepsilon$ for all $\nu$.

Proof. In the following proof, and in $\S 9$, the symbols $K, K_{\varepsilon}$ do not necessarily denote the same constants at each occurrence. In Lemma 4 , choose $s_{0}=\frac{1}{2} P, s_{\nu}=S_{\nu}$ for $\nu \neq 0$. For $|\nu| \geqq 1$, we have $\left|s_{\nu}\right|>\frac{3}{4}$. By Lemma 4 (with $\delta=\min (1 / 2 P, 3 / 4)$ ),

$$
|\psi(z)|<K(1+|z|)^{4 P} e^{\pi|i m z|} .
$$


Now

$$
\Psi(z)=-\frac{P}{2}\left(\frac{z-S_{0}}{z-s_{0}}\right) \psi(z)
$$

and $\left|\left(z-S_{0}\right) /\left(z-s_{0}\right)\right|<K$ for $\left|z-s_{0}\right| \geqq 1 / 4$. For $\operatorname{such} z$, (14) follows from (16). Finally, $|\Psi(z)| \leqq K$ inside $\left|z-s_{0}\right| \leqq 1 / 4$ since this is true on the boundary. This proves (14).

Let $\left|z-S_{\nu}\right| \geqq \varepsilon$ for all $\nu$. If $\left|z-s_{0}\right| \geqq \varepsilon$ then

$$
|\psi(z)|^{-1}<K_{\varepsilon}(1+|z|)^{4 P} e^{-\pi|i m z|}
$$

by Lemma 4 , and $\left|\left(z-s_{0}\right) /\left(z-S_{0}\right)\right|<K_{\varepsilon}$ so that (15) follows from (17) and (18). If, however, $\left|z-s_{0}\right|<\varepsilon$, then for small $\varepsilon$ the disc $\Delta:\left|z-s_{0}\right|<\varepsilon$ is outside each disc $\left|z-S_{\nu}\right|<\varepsilon(\nu= \pm 1, \pm 2, \cdots)$. If it is outside the disc $\Delta^{\prime}:\left|z-S_{0}\right|<\varepsilon$, then $(\Psi(z))^{-1}$ is regular in $\Delta$ and so $|\Psi(z)|^{-1} \leqq K_{\varepsilon}$ in $\Delta$ since this is true on the boundary. If $\Delta$ meets $\Delta^{\prime}$ we apply this argument to the portion of $\Delta$ which is outside $\Delta^{\prime}$.

9. Proof of Lemma 5. By the hypothesis (of Theorem 3), there are positive numbers $P, Q$ such that $\left|\alpha_{\nu}\right| \leqq P<1 / 4,\left|\beta_{\nu}\right| \leqq Q$, for all $\nu$. Let $C_{n}$ denote the rectangular contour whose vertices are $\pm(n+$ $1 / 2) \pm n i$. Let

$$
G(z)=\left(z-l_{0}\right) \prod_{1}^{\infty}\left(1-\frac{z}{l_{\nu}}\right)\left(1-\frac{z}{l_{-\nu}}\right) .
$$

We define

$$
w_{\nu}=\frac{1}{2 \pi i} \int_{-\infty}^{\infty} \frac{G(u) \varphi(u)}{G^{\prime}\left(l_{\nu}\right)\left(u-l_{\nu}\right)} d u
$$

where

$$
\varphi(u)=\frac{1-\cos \pi u}{u} .
$$

Then

$$
\begin{aligned}
\sum_{|\nu| \leqq n} w_{\nu} e^{i l \nu x} & =\frac{1}{4 \pi^{2}} \int_{-\infty}^{\infty} G(u) \varphi(u) d u \int_{\sigma_{n}} \frac{e^{i \zeta x}}{G(\zeta)(u-\zeta)} d \zeta \\
& -\frac{1}{4 \pi^{2}} \int_{-\infty}^{\infty} \varphi(u) e^{i u x} d u \int_{\sigma_{n}} \frac{d_{\zeta}}{u-\zeta} .
\end{aligned}
$$

The last term is

$$
\begin{aligned}
\frac{1}{2 \pi i} \int_{-(n+1 / 2)}^{n+1 / 2} \varphi(u) e^{i u x} d u & =\frac{1}{2 \pi} \int_{-(n+1 / 2)}^{n+1 / 2} \frac{1-\cos \pi u}{u} \sin u x d u \\
& \rightarrow \frac{1}{2} \operatorname{sgn} x
\end{aligned}
$$


boundedly within $(-\pi, \pi)$. Hence it suffices to prove that $I_{n}(x) \rightarrow 0$ boundedly within $(-\pi, \pi)$, where

$$
I_{n}(x)=\int_{-\infty}^{\infty} G(u) \varphi(u) d u \int_{\sigma_{n}} \frac{e^{i \zeta^{x}}}{G(\zeta)(u-\zeta)} d \zeta .
$$

Since $G(z)$ is a function $\Psi(z)$, we have by (15), $|G(\zeta)|^{-1}<K n^{4 P} e^{-\pi n}$ on the horizontal sides of $C_{n}$. Further,

$$
\left|e^{i \zeta x}\right| \leqq e^{n|x|}, \quad|u-\zeta|^{-1}<K(1+|u|)^{-1}, \quad|\varphi(u)|<K(1+|u|)^{-1} .
$$

Since $|G(u)|<K(1+|u|)^{4 P}$ by (14), the contribution to $I_{n}$ of a horizontal side of $C_{n}$ does not exceed in absolute value

$$
K n^{1+4 P} e^{-n(\pi-|x|)} \int_{-\infty}^{\infty} \frac{d u}{(1+|u|)^{2-4 P}},
$$

and tends to zero uniformly within $(-\pi, \pi)$. It remains to consider the contribution to $I_{n}$ of a vertical side of $C_{n}$, say the right side. This contribution is

$$
\begin{aligned}
J_{n}(x) & =\int_{-\infty}^{\infty} G(u) \varphi(u) d u \int_{-i n}^{i n} \frac{e^{i x(n+1 / 2+\zeta)}}{G\left(n+\frac{1}{2}+\zeta\right)\left(u-n-\frac{1}{2}-\zeta\right)} d \zeta \\
& =e^{i x(n+1 / 2)} \int_{-\infty}^{\infty} G\left(u+n+\frac{1}{2}\right) \varphi\left(u+n+\frac{1}{2}\right) d u \\
& \times \int_{-i n}^{i n} \frac{e^{i x \zeta}}{G\left(n+\frac{1}{2}+\zeta\right)(u-\zeta)} d \zeta .
\end{aligned}
$$

For all $\nu$, we define $l_{\nu}^{\prime}=-n+l_{\nu+n}$. Then

$$
\begin{aligned}
\frac{G(z)}{G(w)} & =\frac{\left(z-l_{0}\right)}{\left(w-l_{0}\right)} \prod_{1}^{\infty} \frac{\left(z-l_{\nu}\right)\left(z-l_{-\nu}\right)}{\left(w-l_{\nu}\right)\left(w-l_{-\nu}\right)} \\
& =\frac{z-n-l_{0}^{\prime}}{w-n-l_{0}^{\prime}} \prod_{1}^{\infty} \frac{\left(z-n-l_{\nu-n}^{\prime}\right)\left(z-n-l_{-\nu-n}^{\prime}\right)}{\left(w-n-l_{\nu-n}^{\prime}\right)\left(w-n-l_{-\nu-n}^{\prime}\right)} \\
& =\frac{G_{n}(z-n)}{G_{n}(w-n)}
\end{aligned}
$$

where

$$
G_{n}(z)=\left(z-l_{0}^{\prime}\right) \prod_{1}^{\infty}\left(1-\frac{z}{l_{\nu}^{\prime}}\right)\left(1-\frac{z}{l_{-\nu}^{\prime}}\right)
$$

and $l_{\nu}^{\prime}=\nu+\alpha_{\nu}^{\prime}+i \beta_{\nu}^{\prime}, \alpha_{\nu}^{\prime}=\alpha_{\nu+n}, \beta_{\nu}^{\prime}=\beta_{\nu+n}$. Then $\left|\alpha_{\nu}^{\prime}\right| \leqq P,\left|\beta_{\nu}^{\prime}\right| \leqq Q$. Hence $G_{n}(z)$ is a function $\Psi(z)$ (of Lemma 6) and satisfies the inequalities (14), (15) with constants $K, K_{\varepsilon}$ independent of $n$. In (19), we use the equation 


$$
\frac{G\left(u+n+\frac{1}{2}\right)}{G\left(\zeta+n+\frac{1}{2}\right)}=\frac{G_{n}\left(u+\frac{1}{2}\right)}{G_{n}\left(\zeta+\frac{1}{2}\right)} .
$$

It follows that

$$
\left|J_{n}(x)\right| \leqq \int_{-\infty}^{\infty}\left|G_{n}\left(u+\frac{1}{2}\right)\right| \varphi\left(u+n+\frac{1}{2}\right)|J| d u
$$

where

$$
J=\int_{\gamma} \frac{e^{i x \zeta}}{G_{n}\left(\zeta+\frac{1}{2}\right)(u-\zeta)} d \zeta
$$

and $\gamma$ denotes the path from - in to in modified by replacing the segment $(-i / 8, i / 8)$ by the right half or the left half of the circle $|\zeta|=1 / 8$, according as $u<0$ or $u>0$. On $\gamma, r e(\zeta+1 / 2)$ is between $3 / 8$ and $5 / 8$, and therefore $\zeta+1 / 2$ is at a distance greater than $1 / 8$ from all the zeros of $G_{n}(z)$. By Lemma $6,\left|G_{n}(\zeta+1 / 2)\right|^{-1}<K e^{-\pi|\eta|}(1+|\eta|)$, where $\eta=i m \zeta$. Further $|u-\zeta|^{-1}<K(1+|u|)^{-1}$, and so

$$
\begin{aligned}
|J| & <\frac{K}{1+|u|} \int_{-\infty}^{\infty} e^{-|\eta|(\pi-|x|)}(1+|\eta|) d \eta \\
& <\frac{K}{(1+|u|)(\pi-|x|)^{2}} .
\end{aligned}
$$

Since $\left|G_{n}(u+1 / 2)\right|<K(1+|u|)^{4 P}$, it remains to prove that $H_{n} \rightarrow 0$ where

$$
H_{n}=\int_{-\infty}^{\infty} \frac{d u}{(1+|u|)^{d}\left(1+\left|u+n+\frac{1}{2}\right|\right)}
$$

and $d=1-4 P>0$.

If $m$ is a positive integer, then

$$
H_{n}=\int_{|u| \leqq m}+\int_{|u|>m}
$$

and the first integral tends to zero as $n \rightarrow \infty$. Choose $p$ so that $p d>1$ and let $q^{-1}+p^{-1}=1$. Then

$$
\begin{aligned}
\int_{|u|>m} & \leqq\left(\int_{|u|>m} \frac{d u}{(1+|u|)^{p d}}\right)^{1 / p}\left(\frac{d u}{\int_{-\infty}^{\infty}\left(1+\mid u+n+\frac{1}{2}\right)^{q}}\right)^{1 / q} \\
& <K m^{1 / p-d},
\end{aligned}
$$


so that $\varlimsup H_{n}=0$, as required.

Added in proof. A result similar to Theorem 2 was proved in a $\mathrm{Ph}$. $\mathrm{D}$ thesis by J. A. Anderson.

\section{REFERENCES}

1. J. Korous, On a generalization of Fourier series, Časopis Pěst. Mat. 71 (1946), $1-15$.

2. N. Levinson, Gap and density theorems, (New York 1940).

3. S. Verblunsky, On a class of infinite products, Proc. Cambridge Phil. Soc. 60 (1964), 847-854. 


\section{PACIFIC JOURNAL OF MATHEMATICS}

EDITORS

H. SAMELSON

Stanford University

Stanford, California

R. M. BLUMENTHAL

University of Washington

Seattle, Washington 98105
*J. DUGUNDJI

University of Southern California

Los Angeles, California 90007

RICHARD ARENS

University of California

Los Angeles, California 90024

\section{ASSOCIATE EDITORS}

E. F. BECKENBACH

B. H. NeumanN

F. WOLF

K. YosidA

\section{SUPPORTING INSTITUTIONS}

UNIVERSITY OF BRITISH COLUMBIA

CALIFORNIA INSTITUTE OF TECHNOLOGY

UNIVERSITY OF CALIFORNIA

MONTANA STATE UNIVERSITY

UNIVERSITY OF NEVADA

NEW MEXICO STATE UNIVERSITY

OREGON STATE UNIVERSITY

UNIVERSITY OF OREGON

OSAKA UNIVERSITY

UNIVERSITY OF SOUTHERN CALIFORNIA
STANFORD UNIVERSITY

UNIVERSITY OF TOKYO

UNIVERSITY OF UTAH

WASHINGTON STATE UNIVERSITY

UNIVERSITY OF WASHINGTON

AMERICAN MATHEMATICAL SOCIETY CHEVRON RESEARCH CORPORATION

TRW SYSTEMS

NAVAL ORDNANCE TEST STATION 


\section{Pacific Journal of Mathematics}

\section{Vol. 16, No. $1 \quad$ November, 1966}

Larry Armijo, Minimization of functions having Lipschitz continuous first

partial derivatives ............................... 1

Edward Martin Bolger and William Leonard Harkness, Some

characterizations of exponential-type distributions.............. 5

James Russell Brown, Approximation theorems for Markov operators ...... 13

Doyle Otis Cutler, Quasi-isomorphism for infinite Abelian p-groups ...... 25

Charles M. Glennie, Some identities valid in special Jordan algebras but not valid in all Jordan algebras .......................... 47

Thomas William Hungerford, A description of $\operatorname{Mult}_{i}\left(A^{1}, \cdots, A^{n}\right)$ by

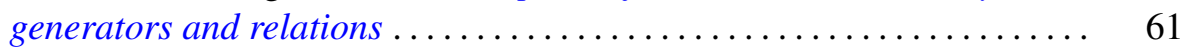

James Henry Jordan, The distribution of cubic and quintic non-residues ....

Junius Colby Kegley, Convexity with respect to Euler-Lagrange differential operators................................... 87

Tilla Weinstein, On the determination of conformal imbedding ......... 113

Paul Jacob Koosis, On the spectral analysis of bounded functions ........ 121

Jean-Pierre Kahane, On the construction of certain bounded continuous functions ................................... 129

V. V. Menon, A theorem on partitions of mass-distribution ........... 133

Ronald C. Mullin, The enumeration of Hamiltonian polygons in triangular

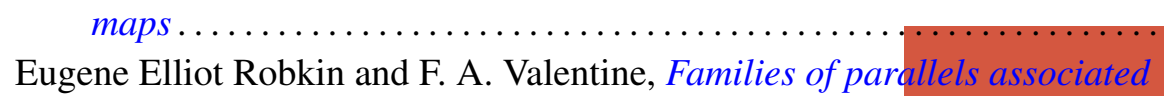

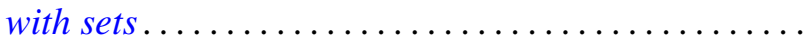

Melvin Rosenfeld, Commutative F-algebras

A. Seidenberg, Derivations and integral closure

S. Verblunsky, On the stability of the set of exponents of a Cauchy

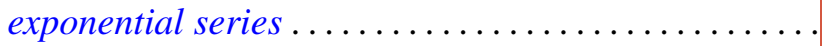

Herbert Walum, Some averages of character sums 